Bull. Mater. Sci., Vol. 4, No. 5, December 1982, pp. 569-573. (Printed in India.

\title{
Deformation study of anhydrous diglycine sulphate crystal
}

\author{
G R PANDYA, D D VYAS and C F DESAI \\ Department of Physics, Faculty of Science, M.S. University of Baroda, \\ Baroda 390 002, India
}

MS received 11 January 1982 ; revised 20 April 1982

\begin{abstract}
The nature of deformation of anhydrous diglycine-sulphate has been stidied. Static and dynamic indentations were employed to deform the crystal. The slip and fracture modes of deformation occurring in the crystal have been identified.
\end{abstract}

Keywords. Deformation ; diglycine-sulphate; static indentation; dynamic indentation ; fracture.

\section{Introduction}

Anhydrous diglycine sulphate (DGs) is one of the phases that crystallizes from aqueous solution of glycine-sulphate (Pandya and Vyas 1980). It has an orthorhombic structure (Wood and Holden 1957) and exhibits perfect cleavage along the (010) plane. Unlike triglycine sulphate, it is a non-ferroelectric crystalline phase of glycine-sulphate and so far very little work has been done on it. Whipps et al (1972) reported the growth and dielectric parameters of the crystal. However, no information on the nature of deformation of this crystal is available. This aspect has now been studied using indentation methods. The slip and fracture modes of deformation occurring in this crystal have been identified.

\section{Experimental}

The DGS crystals used were grown from aqueous solution of glycine-sulphate by isothermal evaporation (Pandya and Vyas 1981). The static and dynamic indentation methods were employed to deform the crystal. A glass rod with a spherical tip ( $3 \mathrm{~mm}$ diameter) was used as the indentor. The indentor rod was guided through a glass tube of suitable diameter fixed vertically on a stand. For static indentation, a horizontal platform to carry the load was fixed to the indentor rod. The loads used range from 200 to $1000 \mathrm{~g}$. The loaded indentor was gradually lowered to the specimen surface. The applied stress was kept normal to the crystal surface. The indentations were carried out on the cleavage plane (010) except otherwise stated. In dynamic indentation, the indentor was allowed to 
fall freely through the glass tube on to the crystal surface. For indentations at high temperatures, a small resistance furnace was used in which the specimen was slowly heated to the desired temperature and was indented after sufficient time interval to achieve thermal equilibrium. The indentor tip was also preheated to the same temperature to avoid thermal shock. The deformed specimens were observed under optical microscope and dislocation etching was carried out for delineating slip.

\section{Discussion}

\subsection{Room temperature deformation}

It was found that the indentations at room temperature produce cracks and the specimen usually underwent brittle type fracture at high loads. No visible traces of slip or twinning were observed. Dynamic indentation at room temperature also caused fracture. Dislocation etching of the indented specimens showed no evidence of slip. Thus the deformation at room temperature did not involve slip or twinning to any significant extent and the crystal shows brittle behaviour.

\subsection{High temperature deformation}

In the high temperature experiments, slip is activated only around $90^{\circ} \mathrm{C}$ and above in static indentation. To reveal the slip, the dislocation etchant consisting of dichloroacetic acid was used (Pandya and Vyas 1981). Figure 1 shows a part of the etch-pit rosette around the indentation mark and there are two prominent rosette wings indicating two distinct slip systems. The rosette arms were parallel to [100] and [101] directions (figure 1). Similar static indentations and etching were carried out on two more planes other than (010), viz (100) and (001). On both the planes the slip traces run parallel to the [010] direction. The slip traces analysed on (010), (100) and (001) planes indicated the system to be (001) [010] and (101) [010]. Out of these, the slip system (001) [010] seems to be more favourably operative than the other. The slip traces corresponding to this system are along [100] direction and in almost all the indentation results, they are in larger number than the [101] traces (figure 1). Hence this system can be recognized as the primary slip system. However, it is interesting to note that the activation of primary and secondary slips shows a dependence on the time for which the load is maintained. Under a given load, the secondary slip occurs after a longer time than that required for the primary slip. Moreover, the density of dislocation pits around the site of indentation increased with the load, temperature and duration of indentation. The time-dependent behaviour of deformation by slip is probably indicative of creep phenomenon associated with it.

Apart from slip, microcracks also accompany the indentation mark to accommodate the deformation. Under high loads, the specimen undergoes fracture. The cracks and fracture are similar in nature as in room temperature indentations. A typical pattern of cracks around the indentation mark is shown in figure 2. The crystallographic orientations of the cracks are noteworthy. In most crack patterns, one main crack was centrally nucleated and was quite straight 


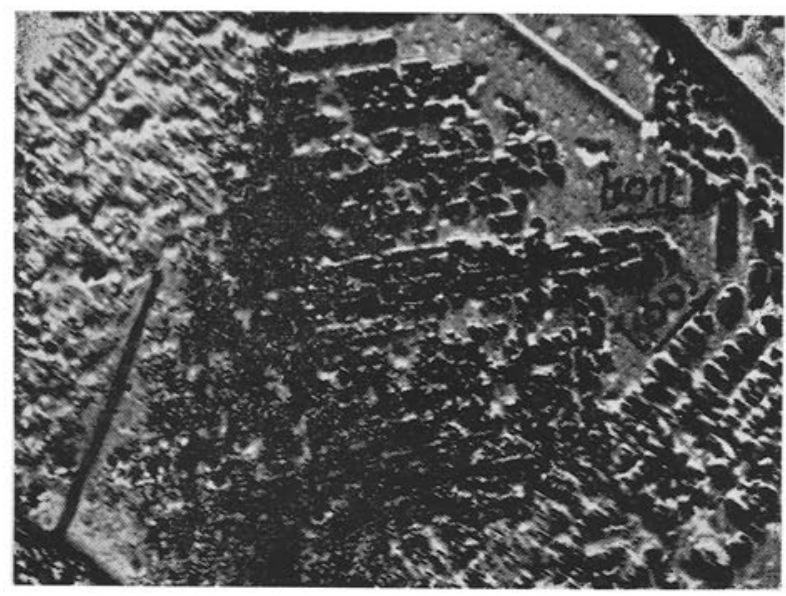

(1)

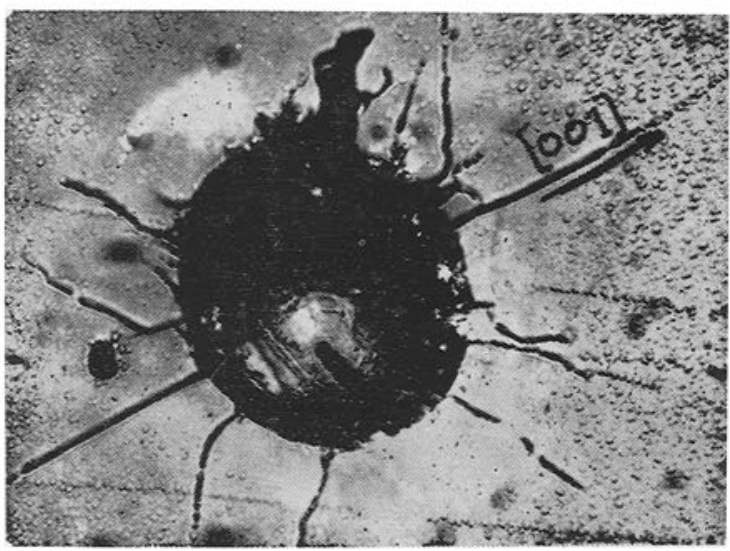

(2)

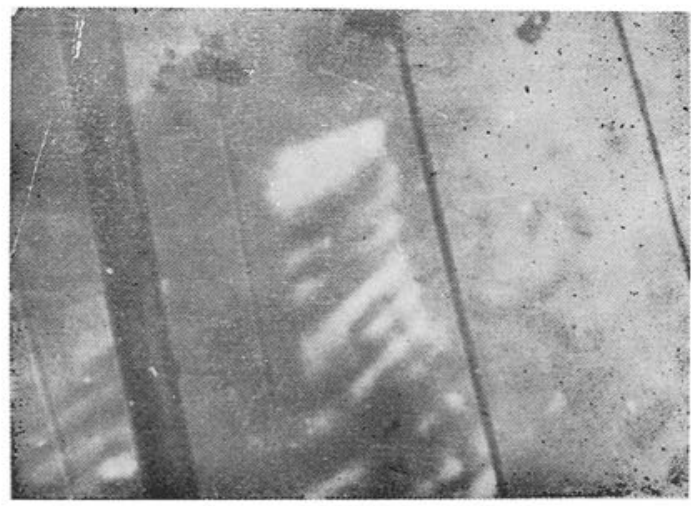

(3)

Figures 1-3. 1. Etch-pit rosette near the indentation mark on the cleaved plane of DGS ciystal $(\times \mathbf{4 0 3 )}$. 2. Static indentation mark on the cleaved DGs crystal $(\times 150)$. 3. Crack lines on the $(010)$ plane running along [001] direction, produced by thermal stress $(x 100)$. 
and along [001] direction (figure 2). It can also be seen that although the other cracks run along different directions like [101] and [201], their initial orientation emanating from the indentation mark is similar to the main central crack. These features indicate that all cracks are nucleated on the same crystallographic plane. In a large number of specimens the fracture occurred along the direction of the main central crack. The fracture surface was identified as [100] by stereographic analysis, implying [001] to be the plane of crack nucleation.

Dynamic indentations carried out at high temperatures resulted in similar cracks and fracture as discussed above. However, the slip was quite suppressed in this case and the crystal frequently showed tendency to undergo complete fracture.

To study the effect of thermal stresses, the specimen was slowly heated to $80^{\circ} \mathrm{C}$ and above and subjected to thermal shock by quenching it at room temperature. Fracture was more pronounced in the resulting deformation. Very sharp thermal shock resulted in brittle fracture giving rise to large size (100) fracture surfaces. When the thermal shock was not quite severe (for example, that produced by moderate quenching), the fracture was incomplete, producing a large number of cracks parallel to (001) direction (figure 3).

\section{Conclusions}

DGS crystal has two slip systems, viz., (001) [010]-primary and (101) [010]secondary. These are activated at temperatures above $80^{\circ} \mathrm{C}$. The cracks and fracture in this crystal are of crystallographic nature. The most prominent crack plane is (100).

\section{Acknowledgements}

The authors express their gratitude to Prof. M M Patel and Dr V P Bhatt for their encouragement during the work. The authors also thank the authorities of Bhabha Atomic Research Centre for awarding a research fellowship to one of them (DDV).

\section{References}

Pandya G R and Vyas D D 1980 J. Cryst. Growth 50870

Pandya G R and Vyas D D 1981 Kristall. Tech. 16319

Whipps O W, Cosier R S and Bye K L 1972 J. Mater. Sci. 71476

Wood E A and Holden A N 1957 Acta Crystal. 10145 